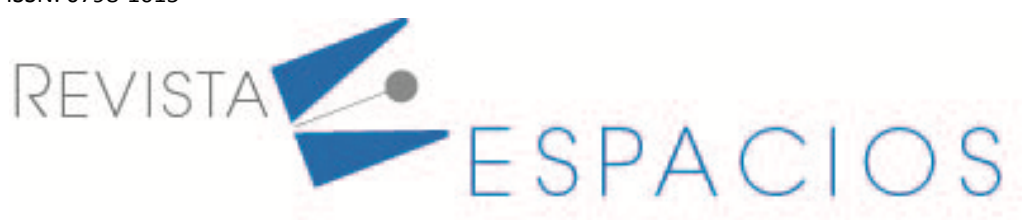

Vol. 41 (49) $2020 \cdot$ Art. 18

\title{
Cualificación del talento humano frente a la organización 4.0 y sus innovaciones
}

\section{Qualification of human talent against the $\mathbf{4 . 0}$ organization and its innovations}

\author{
GONZÁLEZ, Yolanda ${ }^{1}$ \\ MANZANO, Omaira ${ }^{2}$ \\ JIMENEZ, Luis A. ${ }^{3}$
}

\begin{abstract}
Resumen
La investigación propone evaluar las necesidades de cualificación del recurso humano para prepararlo para la organización 4.0, y ofrece un enfoque mixto. En la parte cualitativa presenta una revisión documental, y en la cuantitativa se realiza una investigación no experimental longitudinal de corte descriptivo. Se elaboró un mapa tecnológico evidenciando 8 clúster, cada uno de ellos representa los elementos fundantes de la organización 4.0 y se identifican las competencias que requiere el talento humano para desempeñarse eficientemente en organización 4.0.

Palabras clave: cualificación, talento humano, organización 4.0, innovaciones

Abstract

The purpose of the research was to evaluate the qualification needs of human resources to prepare them for the 4.0 organization. The study responds to a mixed approach. In the qualitative part, a documentary review is carried out and in the quantitative aspect, a descriptive longitudinal nonexperimental investigation is carried out. A technological map was drawn up showing 8 clusters, each of them represents the founding elements of the 4.0 organization and the competencies that human talent requires to perform efficiently in the 4.0 organization are identified.

key words: qualification, human talent, organization 4.0, innovations
\end{abstract}

\section{Introducción}

La Sociedad 4.0 es una plataforma para organizar y exaltar un movimiento de personas para colocar de manera activa y metódica el futuro de la humanidad en manos de la gente. En ese sentido, el rápido desarrollo de las tecnologías de la información y la comunicación (TIC), permite vivir en un mundo digital interconectado, caracterizado por una mayor movilidad y un acceso a la información oportuna. Al mismo tiempo, se aborda una serie de problemas globales, como la mejora de la salud, la protección del medio ambiente y la lucha contra la pobreza. Las partes interesadas, o stakeholders, exigen que las empresas adopten enfoques más transparentes y responsables, con el objetivo holístico de crecimiento económico, progreso social, equidad, respeto y

\footnotetext{
${ }^{1}$ Doctora en Administración de Negocios (DBA). Magíster en Recursos Humanos y Gestión del Conocimiento. Investigadora grupo GRINDES. Docente Asociado, Universidad Nacional Abierta y a Distancia (UNAD). E-mail: yolanda.gonzalez@unad.edu.co

2 Doctora en Administración de Negocios (DBA). Investigadora grupo GRINDES. Docente Asociado, Universidad Nacional Abierta y a Distancia (UNAD), Ocaña - Colombia. E-mail:: omaira.manzano@unad.edu.co

${ }^{3}$ Doctor en Ciencias Gerenciales. Magister en Gestión y Desarrollo en Empresas Sociales. Esp. Control Interno e Indicadores de Gestión. Administrador de Empresas. Docente - Investigador Universidad Nacional Abierta y a Distancia UNAD. E-mail:alfredo.jimenez@unad.edu.co
} 
conciencia del medio ambiente (Fonseca, et al., 2016). Las organizaciones deben hacer frente a estos grupos de interés cada vez más exigentes y responder a estos desafíos y riesgos mientras se aprovechan las oportunidades disponibles.

A propósito, para la UNESCO (2018) la percepción pluralista de sociedades del conocimiento va más allá de la sociedad de la información, ya que encamina a una metamorfosis social, cultural y económica como ayuda al desarrollo sustentable. Se considera la sociedad del saber como legatario de un acumulado de participaciones en el acontecer de la sociedad de la información. Khan (2003) refirió que "la sociedad de la información es la piedra angular de las sociedades del conocimiento". El pensamiento de "sociedad de la información" está conexo con la idea de la "innovación tecnológica", mientras que el concepto de "sociedades del conocimiento" incorpora una capacidad de transformación cultural, económica, social, política e institucional, y una enfoque más diversificado y potenciador.

En una visión evolutiva, puede verse como el sucesor de una fase previa a la sociedad de la información, que a su vez siguió a la sociedad industrial, llamada así debido al gran flujo de información que se activó con la llegada de las computadoras, los sistemas de procesamiento de datos y las comunicaciones. En la sociedad del conocimiento, el conocimiento, y no la mera información, es el activo más valioso, es lo que está en la cabeza de la gente (conocimiento tácito). Así, dado que el conocimiento tácito, a menudo se materializa en personas educadas o en capital humano, cuyo impacto positivo también implica la importancia de una buena educación a nivel micro y macro.

Por consiguiente, la sociedad de la información, constituye una etapa en la formación de una sociedad basada en el conocimiento sin la cual no se puede desarrollar una sociedad duradera. De manera que una sociedad ecológicamente sostenible no puede existir sin una sociedad del conocimiento. Por lo tanto, la sociedad del conocimiento puede ofrecer soluciones para resolver el problema del desastre ecológico que podría ocurrir en un futuro no muy lejano (Bustillo-García y Martínez-Dávila, 2008).

En ese orden, Chandrasekar y Sharma (2010) intentaron explicar el impacto del conocimiento en la actividad económica, y concluyeron que, en el caso del crecimiento y mejora a largo plazo de una sociedad, la diferencia de conocimiento dentro de la sociedad es crucial. Se asumió que el antagonismo de conocimiento dentro de las sociedades se basa en su capacidad para vincular el conocimiento con la creación de valor. En ese sentido, Yigitcanlar (2011) vinculó el concepto de conocimiento con el desarrollo urbano como un paradigma prometedor para apoyar el proceso de transformación de las ciudades en ciudades del conocimiento y sus sociedades en sociedades del conocimiento. Además, discutió el desarrollo de las ciudades futuras destacando particularmente los desafíos y oportunidades potenciales que previamente no se habían considerado completamente.

En ese mismo enfoque, en el medio de evolución de la sociedad industrial a una sociedad de la información se percibe una expedita gestación y un aumento del uso de las tecnologías de la información y de la comunicación, lo cual ejerce un alto impacto sobre todos los aspectos de la vida. En ese sentido, tienden a desaparecer las habituales distinciones entre medios de comunicación, telecomunicaciones, informática y servicios de información.

No obstante, es interesante lo planteado por Kozma y Schank (2000) donde describen que la educación ya no está encapsulada en función del tiempo, lugar y edad, sino que ha pasado a convertirse en una actitud generalizada que continúa durante toda la vida. Si la enseñanza humana consiste en adquirir, procesar, comprender, sintetizar, valorar y aplicar una información que ha sido para el aprendizaje, se puede decir que es una continua sintaxis de conocimiento. Autores como Weingart (2001) y Heidenreich (2003) han deliberado sobre el concepto de la sociedad del conocimiento relacionándolo tanto con la política como con otras ciencias sociales. 
De acuerdo con el análisis anterior, las herramientas de transformación digital más populares de las corporaciones a partir de 2016 son la información y el almacenamiento en la nube, el análisis de Big data, las redes sociales y las aplicaciones móviles. Estas versiones, por poderosas que sean, reflejan la visión limitada de los proveedores y consultores de servicios de tecnología de información. No reflejan los cambios sociológicos, organizativos y de mercado que realmente caracterizan a la sociedad del conocimiento.

Por lo tanto, se considera que la información tiene valor económico y puede utilizarse para promover el desarrollo humano en áreas como la salud, la educación, los servicios sociales y el comercio. Esto conduciría a un aumento de la capacidad intelectual humana y puede conducir a la mejora económica de las condiciones de trabajo y de vida diaria. El uso de las TIC modernas dentro de la sociedad de la información y el conocimiento conducirá a la libertad de información que puede llevar a un proceso político caracterizado por una mayor participación en el campo político de los ciudadanos.

En ese orden, los desarrollos tecnológicos, los adelantos de las telecomunicaciones crearon una infraestructura favorable para el surgimiento de un fenómeno histórico que rige nuestra sociedad, "la globalización", que no es más que un proceso de expansión hasta niveles universales de actividades humanas importantes como las económicas, políticas o culturales que inicialmente se extendían apenas a pocos países, hoy, las conexiones de comunicación llevan la información desde los centros de poder económico, político, científico, hasta los más recónditos lugares del mundo y viceversa. Esa fantástica telaraña comunicacional y una intención deliberada del capitalismo mundial sustenta, no sólo las transacciones casi instantáneas que caracterizan la economía actual, sino que a todas luces fortalecen el poder desconocido de las grandes compañías.

Por lo tanto, el objetivo del presente artículo es realizar un estudio cienciométrico que permita determinar los avances de las organizaciones 4.0 y su clasificación temática de acuerdo con la implementación de la tecnología 4.0. Además, se identifican las competencias que requieren el talento humano para desempeñarse en forma efectiva como parte activa de la sociedad y la organización 4.0. Para lo cual se emplearon las bases de datos Web of Science y Scopus y el software VOSViewer.

\section{Metodología}

La presente investigación responde a un enfoque mixto. En la parte cualitativa se efectúa una revisión documental de diversas bases de datos científicas y en el aspecto cuantitativo se realiza una investigación no experimental longitudinal de corte descriptivo. En este sentido, Hernández, Fernández y Baptista (2014) afirman que el interés del investigador es analizar cambios con el paso del tiempo en determinadas categorías o variables.

Las fases de la investigación son las siguientes:

Fase 1. Análisis de contenidos bibliográficos sobre la evolución de la sociedad del conocimiento y el involucramiento tecnológico hasta lo que hoy se conoce como la sociedad 4.0, con el propósito de identificar sus características

Fase 2. Comprensión de los avances relacionados con las organizaciones 4.0 mediante un estudio bibliométrico que permita inferir la evolución de conceptos y teorías involucradas con las competencias disciplinares.

Fase 3. Determinación del papel de la educación superior en el desarrollo de las competencias genéricas y disciplinares requeridas para que los egresados puedan desenvolverse de forma adecuada en una sociedad y organización 4.0 . 


\section{Resultados}

Mediante un estudio bibliométrico relacionado con la organización 4.0 es posible identificar lo que actualmente se está haciendo en torno a la cuarta revolución industrial en el ámbito de las empresas y de esta forma poder planear e intervenir en los modelos de negocios con elementos determinantes para ingresar a un mundo avanzado e inteligente. Para determinar los avances de las organizaciones 4.0 y su clasificación temática de acuerdo con la implementación de la tecnología 4.0, se realizó un estudio cienciométrico, utilizando como bases de datos científicas Web of Science y Scopus. La ecuación de búsqueda fue "industria 4.0" OR "industry 4.0" y el software de análisis VOSViewer. En la base de datos aparece un total de 986 resultados de artículos científicos escritos entre 2016 y 2018 sobre el tema de organizaciones o industria 4.0. Los artículos muestran una tendencia creciente sobre el área temática. Se observa un aumento de 5 veces más de publicaciones entre el 2016 y 2018 (Ver figura 1).

\section{Figura 1}

Publicaciones

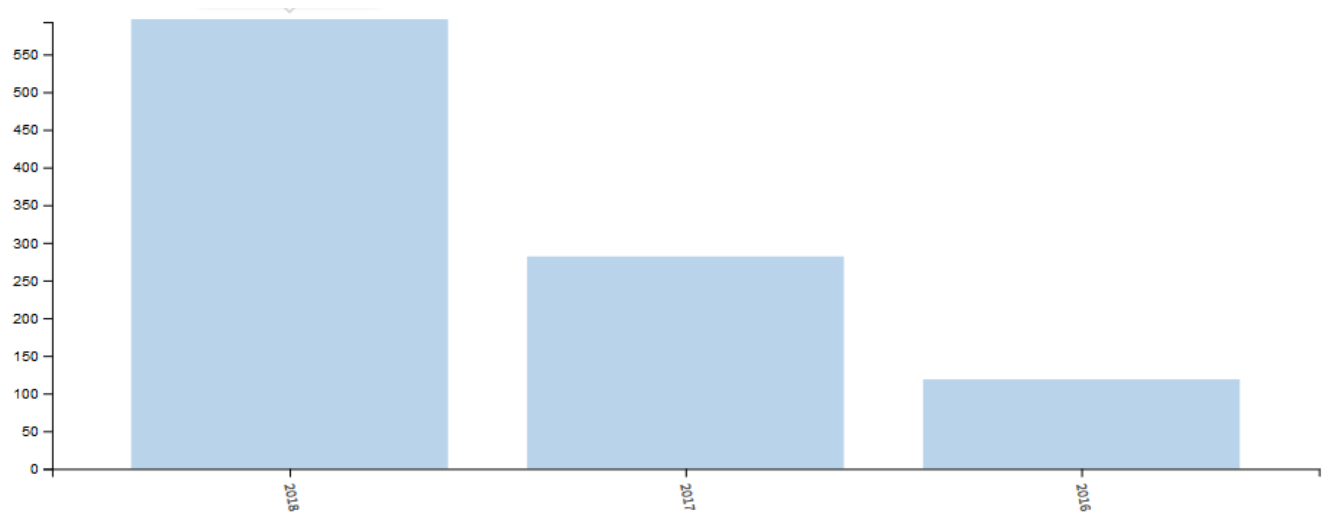

Fuente: Base de datos Web of Science

Las principales fuentes donde los autores publican sus artículos son en las revistas de acceso abierto y de amplia difusión como Procedia Manufacturing y Procedia CIRP y en revistas de tecnologías de avanzada como IFIP Advances in Information and Communication Technology y Lecture Notes in Computer Science (Ver figura 2).

Las instituciones que más publican sobre este tema son: La Universidad Tecnológica del Sur de China, seguida por la Universidad Técnica de Aquisgrán.

En la base de datos de Web of Science un gran número de libros fue publicado por anónimos, en tal sentido se realizó la misma revisión en la base de datos Scopus y se encontró que los autores con mayor número de publicaciones sobre el tema son Rauch, Erwin de la Universidad Libre de Bozen - Bolsano en Italia y Matt, Dominic de la organización de investigación aplicada Fraunhofe (Ver Figura 3). 
Figura 2

Fuentes de publicación

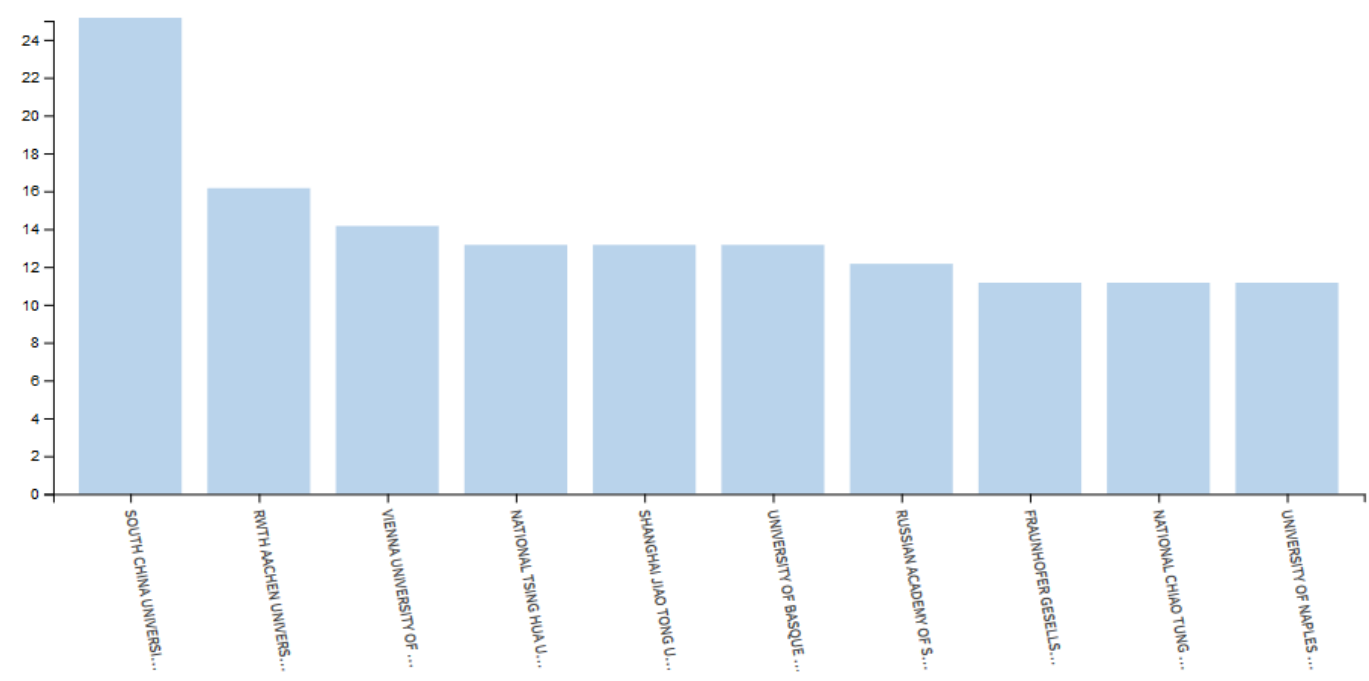

Fuente: Base de datos Web of Science

Figura 3

Número de documentos por autor

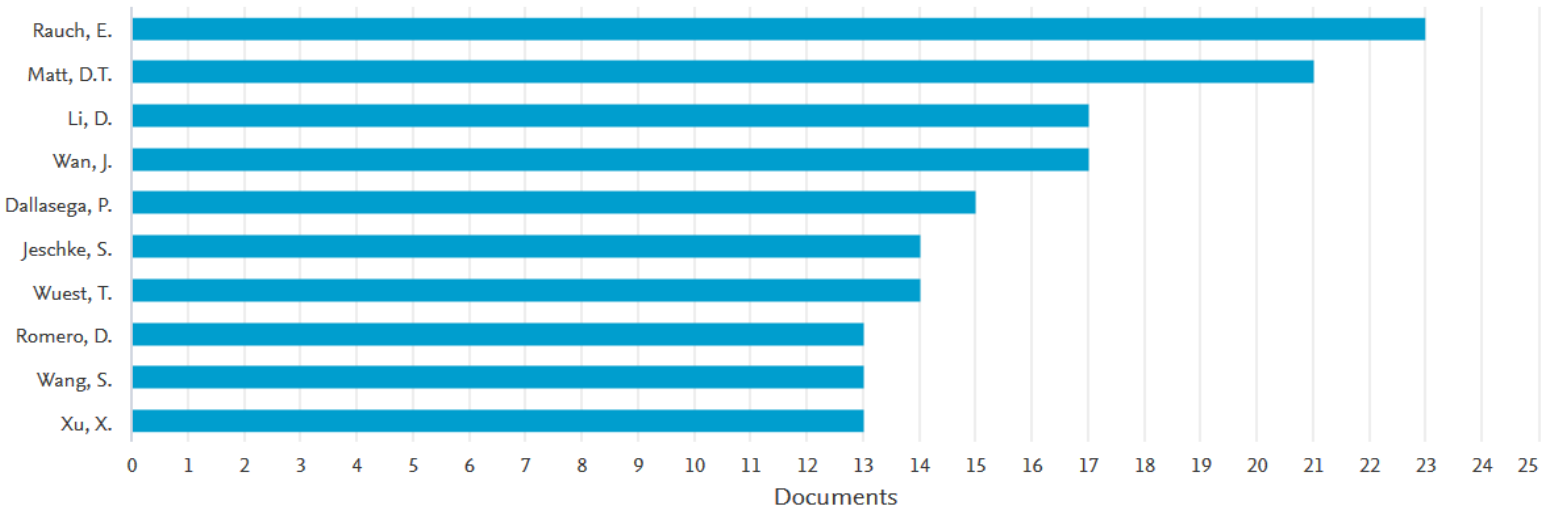

Fuente: Base de datos Scopus

Los países que más publicaciones han realizado sobre la temática son: Alemania, seguido de China, España, Italia y Estados Unidos (Ver figura 4). 
Figura 4

Países con más

publicaciones 2016-2018

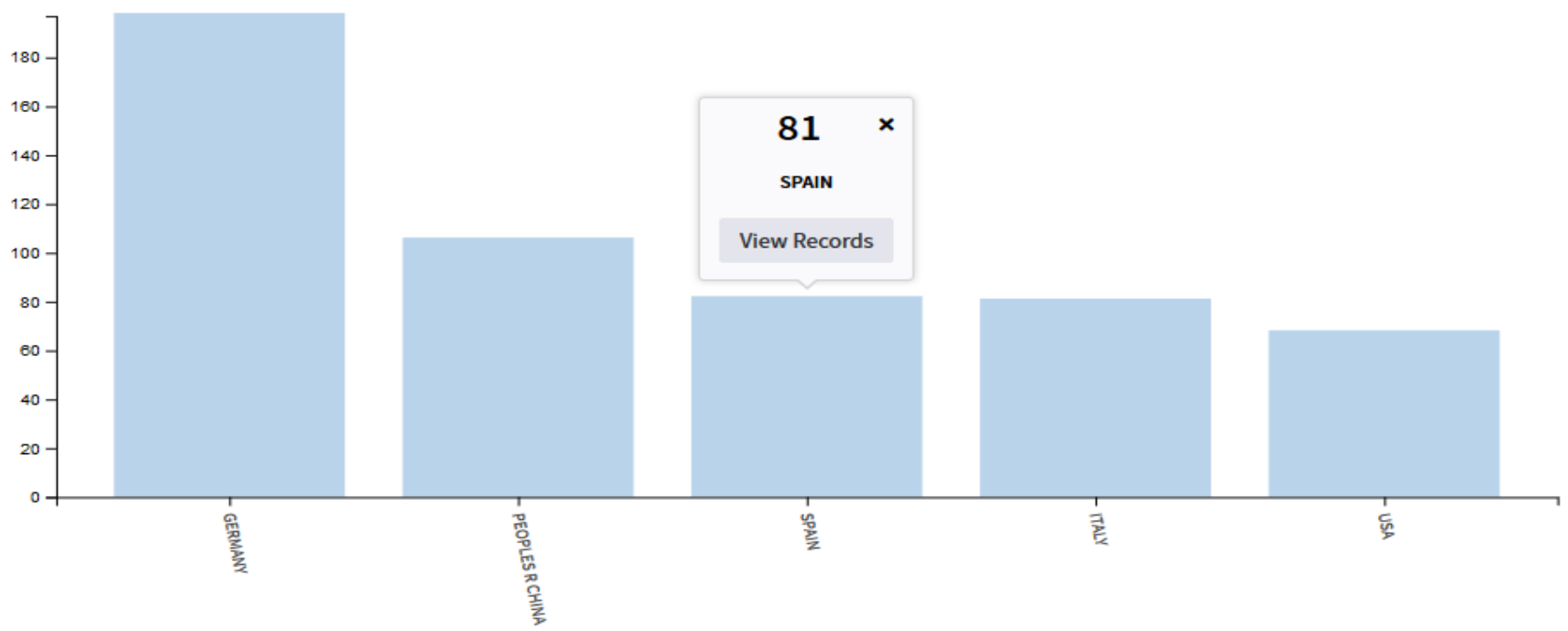

Fuente: Base de datos Web of Science

El 30,4\% de los documentos publicados sobre industria 4.0 son del área de ingeniería en un $47 \%$, seguido de las ciencias computacionales con un $25 \%$, sistemas de automatización y control con un $12 \%$ y economía y negocios con el $11 \%$ (Ver Figura 5)

Figura 5

Temáticas con más publicaciones

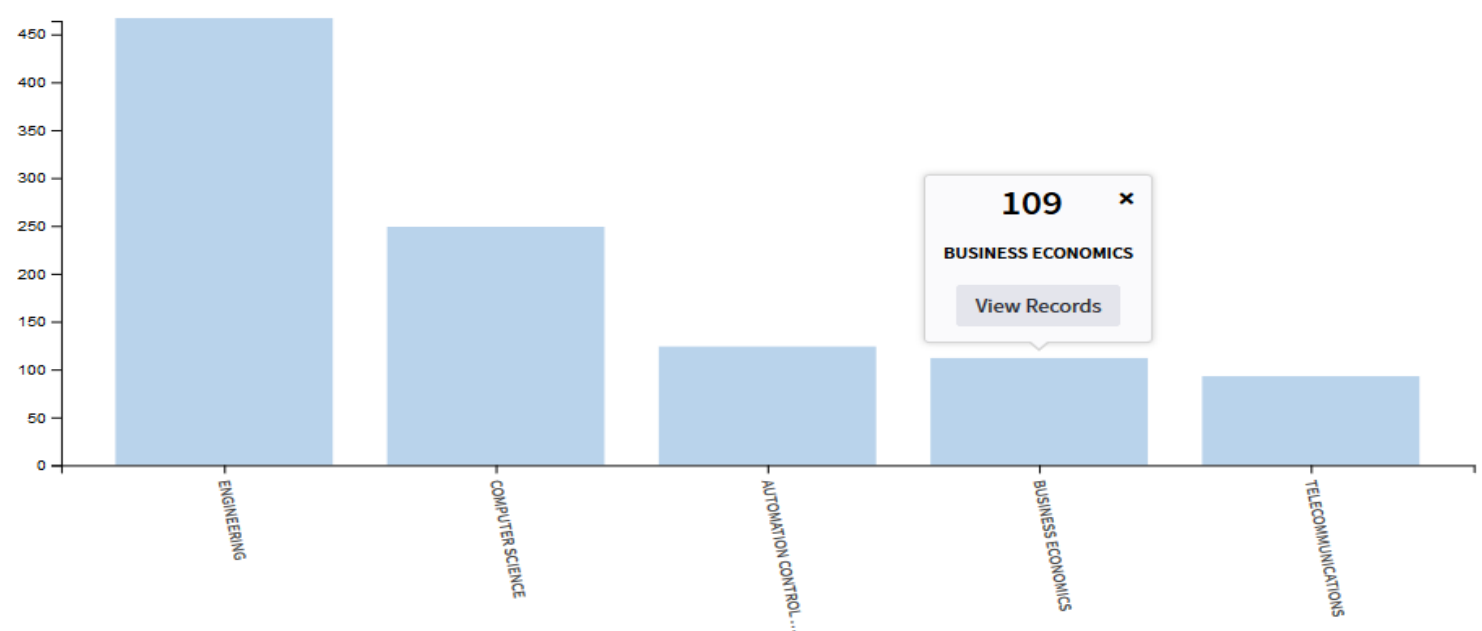

Fuente: Base de datos Web of Science.

La socialización del conocimiento se realiza por medio de artículos en más de un 82\% (Ver Figura 6). 
Figura 6

Difusión del conocimiento.

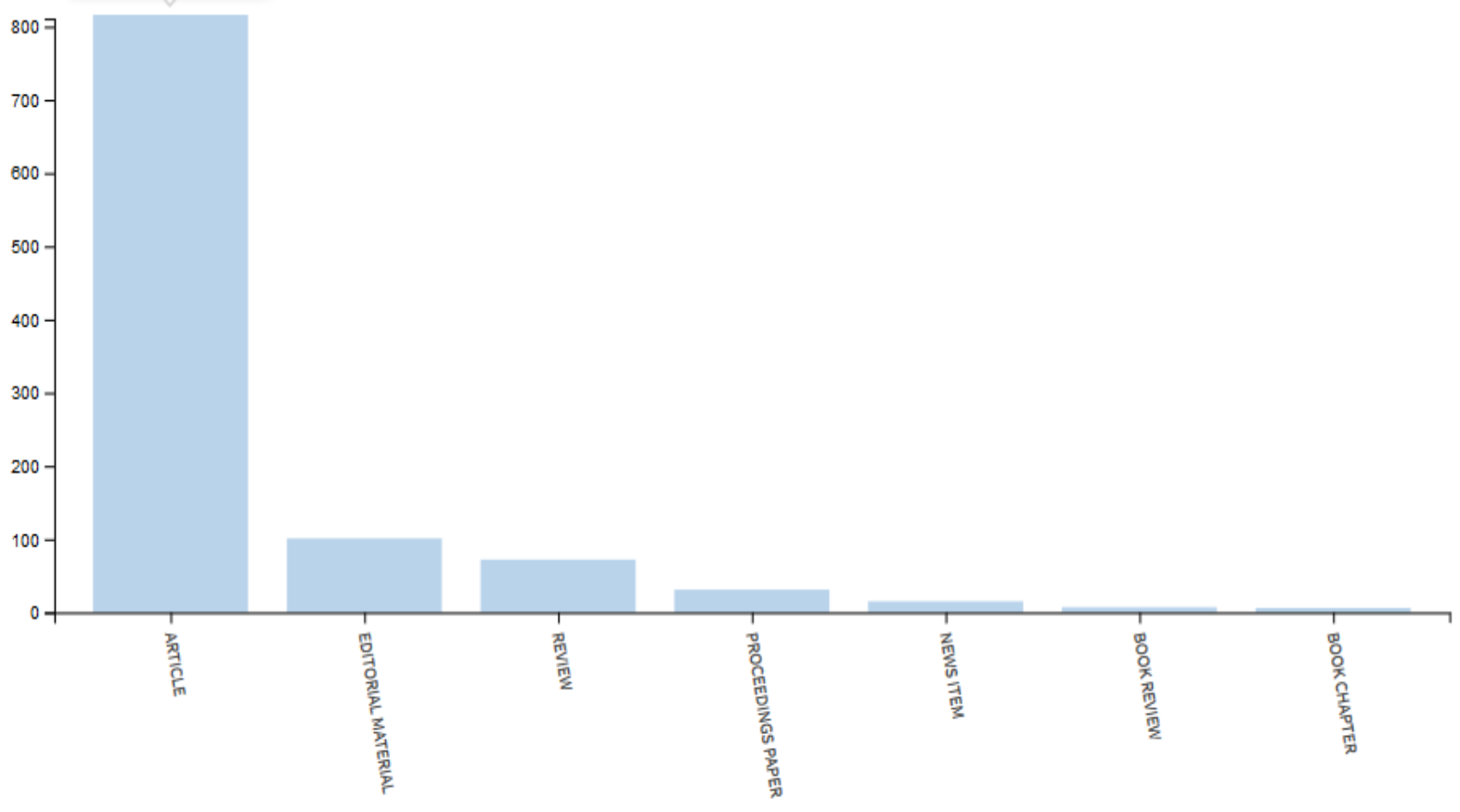

Fuente: Base de datos Web of Science.

De la bibliometría se puede evidenciar como la industria u organización 4.0 se ha venido desarrollando en forma vertiginosa en los últimos años, y la difusión del conocimiento se materializa utilizando ponencias y artículos de rápida difusión por la acelerada innovación que este tema representa. Latinoamérica no tiene un lugar avanzado en la producción científica sobre el tema, pero tiene mucho por aprender de países como Alemania, China y España. La producción científica en organización 4.0 se produce en gran medida desde las ciencias ingenieriles, no obstante, se evidencia que desde las ciencias económicas y administrativas ya emerge un interesante nivel de productividad.

Con el propósito de identificar los indicadores o subtemas que han dado lugar a publicaciones científicas relacionadas con las organizaciones 4.0 se elaboró un mapa tecnológico mediante el uso del software VOSViewer. En el gráfico resultante se evidencian 8 clúster, cada uno de ellos representa los elementos fundantes de la organización 4.0 y ellos son: Innovación, logística, comunicación, internet de las cosas, sistemas automáticos y robótica, producción, evaluación y control, nuevas tecnologías en sistemas de información y analítica de datos (Ver Figura 7). 


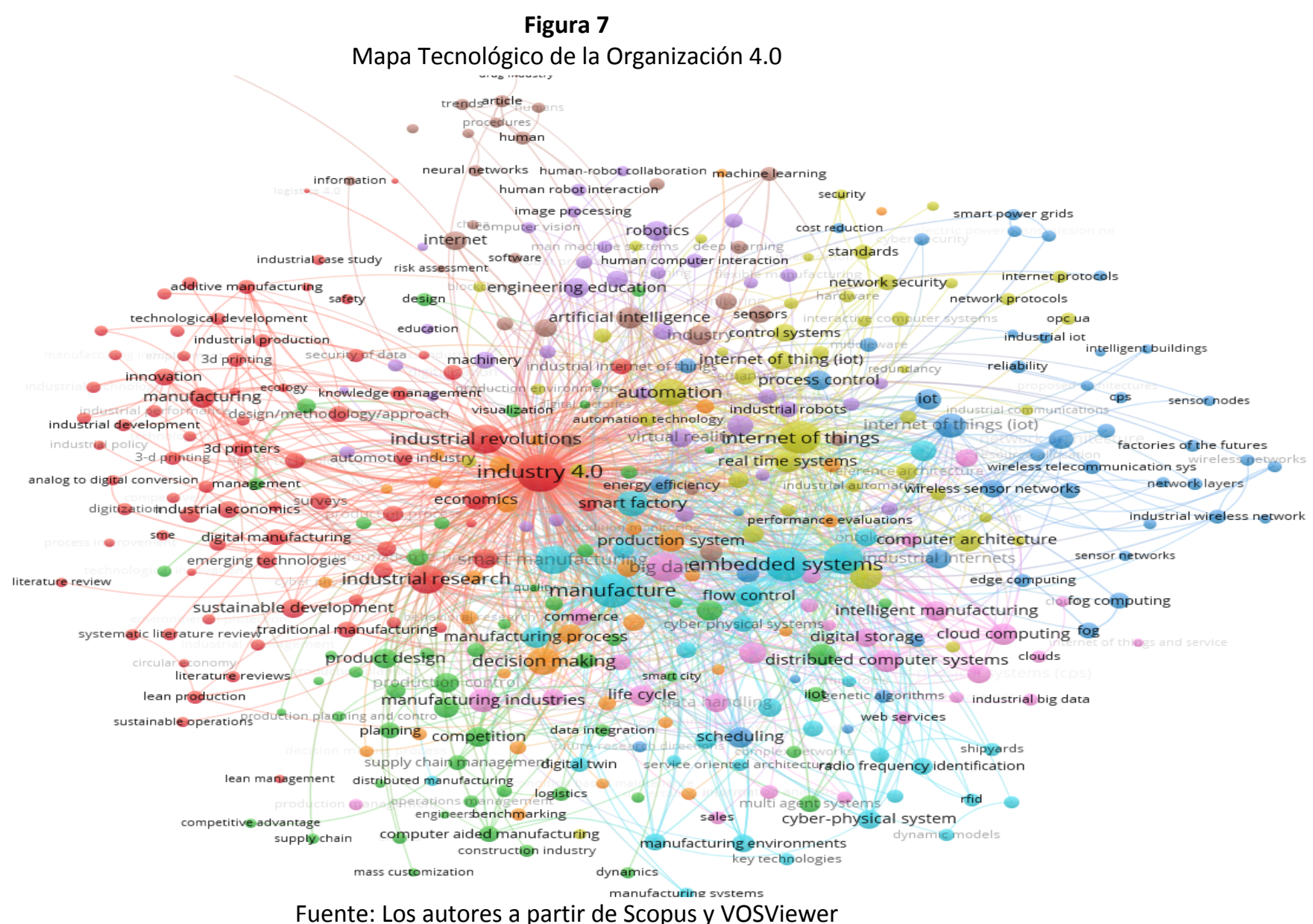

Fuente: Los autores a partir de Scopus y VOSViewer

Nota: Los clústeres se diferencian de acuerdo con los colores de la siguiente forma:

1. Rojo: Innovación;

2. verde oscuro: logística;

3. azul claro: telecomunicaciones;

4. verde claro: internet de las cosas;

5. Lila: sistemas automáticos y robótica;

6. Naranja: producción, evaluación y control;

7. Café: nuevas tecnologías en sistemas de información y control;

8. Morado: analítica de datos

La innovación. Integrar, innovar y desarrollar procesos autónomos es la carta de presentación de las organizaciones 4.0, se trata de transformar sus propios modelos de negocios al integrar el entorno y las tecnologías a la organización. La innovación permite la creación de productos y empleos diversos y es uno de los pasos para lograr la transformación de las empresas en organizaciones inteligentes (Ynzunza, Izar, Bocaranda, Aguilar y Larios, 2017). Existen diversas formas de innovar como la disruptiva, donde se integran las tecnologías, la investigación y la implementación de nuevas estrategias sostenibles de mercado (Restrepo, 2013). Así también, está la denominada innovación abierta, traducida en cooperación con externos y en red para encontrar soluciones comunes a situaciones de cambio específicas, utilizando las competencias internas que posee cada organización (Jamett, Alvarado \& Maturana, 2017). Ahora bien, la innovación abierta y disruptiva soportada en nuevas arquitecturas tecnológicas se convierte hoy en día en una competencia necesaria para la competitividad (Avendaño, 2012). Actualmente, la integración que ha logrado la digitalización al conectar productos, cosas y personas, evidencia un cambio significativo en los procesos de innovación (Domínguez, 2003). 
Logística. La integración de sistemas tanto físicos como virtuales permiten crear ventajas competitivas a los procesos logísticos, disminuyendo costos y aumentando la efectividad en las entregas. Se trata de aprovechar las fortalezas de los sistemas digitales para transformar datos en información útil, de fortalecer las capacidades del recurso humano y sistematizar los procesos haciendo una reacomodación de tareas para lograr mejores resultados. Es innegable la necesidad de establecer conexiones sistémicas en los procesos para transmitir servicios y productos de un lugar a otro como también información. Así como también, aprovechar las nuevas capacidades para gestionar en mayores niveles de complejidad. (Kagermann, Wahlster \& Helbig, 2013). Un sistema logístico organizado permite cumplir con el justo a tiempo, revaluar los procesos y procedimientos de acuerdo con las necesidades que establece el usuario y que él mismo puede medir en tiempo real (Lee, 2014).

Telecomunicaciónes. Una de las grandes ventajas de la organización 4.0 es el internet y las amplias posibilidades de comunicación real sin importar la distancia (Molina et al., 2019). El avance de las telecomunicaciones para la trasmisión de voz, datos, imágenes e información por redes conduce a nuevas formas de trabajo, al igual que demuestra avances en el aprendizaje organizacional (Díaz, Pérez \& Florido, 2011). Lo anterior aunado a los avances computacionales entregan a las organizaciones herramientas de avanzada para mantenerse actualizadas, liderar procesos, recopilar datos y tomar decisiones en menor tiempo. De igual forma, estas herramientas tecnológicas administradas en forma adecuada pueden proporcionar incluso nexos vivenciales entre las personas (Rueda-López, 2007); y con la digitalización de la industria es posible mantener una comunicación permanente no solo hombre - hombre sino también hombre máquina, así como también enfrentarse a situaciones complejas que demandan desarrollo de la competitividad, coordinación en y entre equipos, toma de decisiones con flexibilidad y armonía para fomentar equipos de trabajo que fusionan sus esfuerzos por objetivos comunes (Rueda-López, 2007).

Internet de las cosas. El internet de las cosas está transformando la internet tradicional abriendo a un nuevo aprovechamiento de las tecnologías (Del Val Roman, 2016). Se trata de la construcción de modelos que trasladen el mundo físico al virtual y controlarlos mediante programas, máquinas, dispositivos y sensores. Con este avance es posible optimizar recursos, racionalizar procesos, así mismo, mediante el internet de las cosas se consigue predecir situaciones antes de que sucedan, y mantener un contacto permanente entre los actores, ya sea para mantenerse actualizados o para dar a conocer las ventajas competitivas que se poseen (Díaz-Martínez, CruzMéndez y Ruíz-Domínguez, 2018).

Algunos ejemplos de la aplicación del Internet de las cosas son los siguientes: Identificar fallas a partir de sensores, controles precisos en los inventarios o de los productos terminados, lograr un conocimiento más amplio de los gustos y preferencias de los usuarios o el diseño de publicidad especializada, adicionalmente todos aquellos relacionados con la industria y el comercio.

Sistemas automáticos, robótica. La robótica es considerada como una de las tecnologías emergentes en el marco de la cuarta revolución industrial, al ser el resultado de la convergencia de numerosas especialidades y técnicas que impactan organizaciones de diversos sectores económicos, al permitir la optimización y eficiencia de procesos, generando aportes a la economía circular (García, 2018). Cada vez es más común encontrar los sistemas automáticos y robots, ya sea en procesos tan complejos como el ensamble de carros, o en tareas sencillas como las de limpieza en el hogar, pasando por robots que dan atención a los consumidores, como los cajeros. Por otra parte, se espera que en el futuro tres de cada cuatro empleos estén relacionados con estas áreas. (Amor Bravo, 2019). Basados en la automatización se conocen ya los robots programados para realizar tareas sin ayuda humana, y empieza a hablarse de la robótica colaborativa, que es la integración de humanos con los robots para desarrollar diferentes actividades en una organización (López, Lovato y Abad, 2018). En este tipo de colaboración los robots son usados para suplir aquellas características que para un ser humano es difícil realizar, como por ejemplo, realizar labores repetitivas sin cansarse como en el caso de empaques de grandes 
cantidades de productos, lograr mayor exactitud en los ensambles de piezas, entre otras. Las actividades de los robots se complementan con los análisis del ser humano en este trabajo conjunto.

Producción, evaluación y control. La cuarta revolución industrial permite un mayor control a la cadena de suministro desde el inicio del abastecimiento hasta la entrega al consumidor final (Diaz-Martinez, Cruz-Méndez y Ruíz-Domínguez, 2018), a la vez que arroja información homogeneizada sobre todo el proceso mejorando la planificación y el desempeño de cada área (Pesce, Rigon, Tregnaghi y Voglino, 2017). La idea de implementar tecnologías 4.0 a los procesos de producción, evaluación y control es generar redes inteligentes de productos al igual que de procesos involucrados en la cadena de valor. Se trata de crear cadenas de valor inteligentes que se realimenten e interpreten los cambios que se requieran para ser más flexibles, que puedan entregarse productos terminados o no terminados para su comercialización en cualquier momento del proceso productivo y especialmente que se aprovechen las capacidades tecnológicas interactuantes para tomar decisiones inteligentes.

Nuevas tecnologías en sistemas de información. El manejo de la información es una de las prioridades para estar a la vanguardia, es por esto por lo que las nuevas tecnologías han cambiado la forma como se accede y se interpreta la información. En la actualidad, las áreas de producción se ven beneficiadas por la incorporación de procesos inteligentes, y el área comercial por la posibilidad de detectar oportunidades de negocio (Pérez, 2018).

Los avances en las tecnologías en sistemas de información han dado lugar a fabricación agregada basada en las impresiones de tercera dimensión para la construcción de maquetas y dispositivos, la inteligencia artificial para utilizar el computador en actividades propias del ser humano, la realidad aumentada con la combinación de elementos físicos con los virtuales y la denominada realidad virtual para entrenamientos de trabajadores. Todas estas tecnologías aplicadas a las organizaciones llevan a los países avanzados al alcance de logros. Algunos ejemplos de estas tecnologías se encuentran en el montaje y diseño de instalaciones, en la preparación a trabajadores para identificar formas de actuar en situaciones complejas.

Analítica de datos. Las estrategias de digitalización en la cuarta revolución industrial están basadas en las tecnologías de analítica de datos y se fundamenta en la gran cantidad de información que actualmente se encuentra almacenada en grandes repositorios para ser utilizada en tiempo real. Esta información y su tratamiento se hace a partir de software especializados garantizando el flujo, procesamiento y seguridad de ellos (Ynzunza et al., 2017). En la aplicación de la analítica de datos para las organizaciones se puede realizar analítica descriptiva como una reflexión del pasado, de diagnóstico para establecer las causas de las situaciones, predictiva para hacer una aproximación al futuro de la organización y prescriptiva para identificar acciones que permitan cambiar el futuro de la organización.

Ahora bien, para conocer el avance de las tecnologías relacionadas con la organización 4.0 se realizó una revisión de patentes y se evidenciaron los siguientes resultados: En términos generales las mayores patentes de tecnologías relacionadas con organizaciones 4.0 son la innovación y el internet de las cosas, seguidos de tecnologías de logística y productividad. Las otras tecnologías son emergentes. 
Figura 8

Patentes existentes con tecnologías

relacionadas con organizaciones 4.0 .

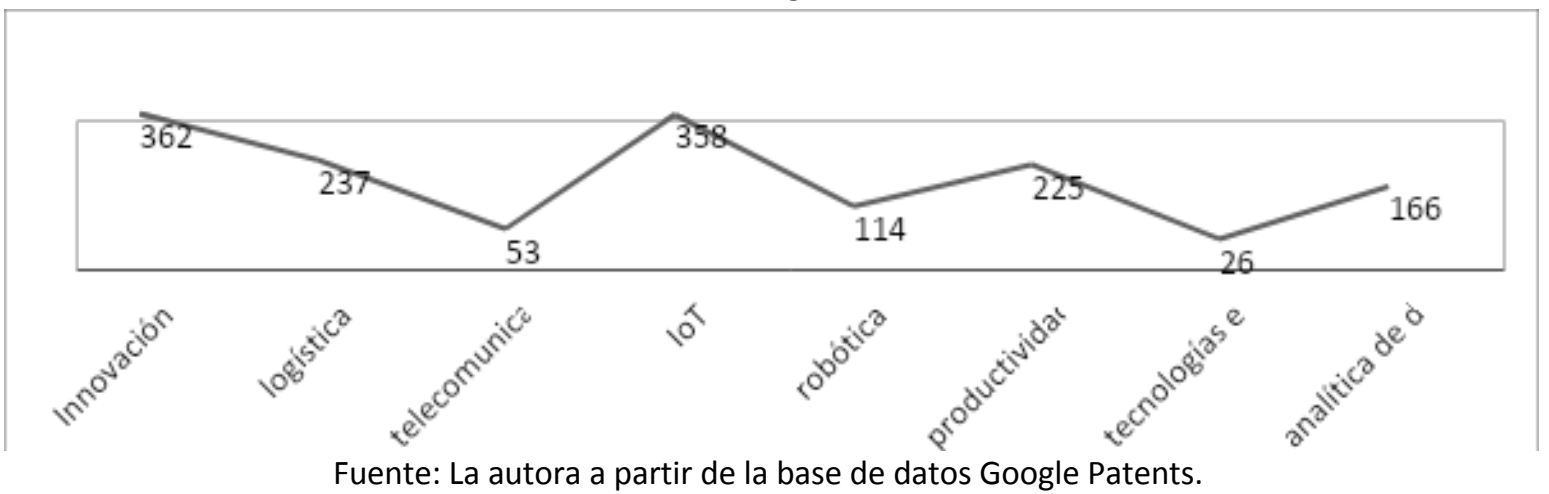

De acuerdo a lo anterior, las organizaciones 4.0 permiten la innovación con la combinación de tecnologías emergentes. Esta innovación constante se ve reflejada en la mejora de la productividad y competitividad. Para que los empresarios puedan tener una mejor idea de cómo otras organizaciones han asociado dichas tecnologías es importante la revisión de bases de datos tanto científicas como de patentes (del Val Román,2016). Su implementación como todo proceso de cambio demanda el respaldo de la alta gerencia, capacitación a todos los miembros de la organización y una reorganización para obtener mejores resultados. Inicialmente será un proceso que demandará tiempo pero una vez adaptado se verán muy pronto los resultados (Ynzuanza, Izar, Aguilar, \& Larios, 2017).

La sociedad 4.0 es el resultado de integrar la gestión del conocimiento de los avances tecnológicos a las características propias de la organización. Su objetivo es transformar la sociedad hacia el crecimiento y desarrollo, situación que implica no solo la rentabilidad de las empresas sino también el bienestar, el trabajo digno y la sustentabilidad (Drath y Horch, 2014). Para ello se requiere que este conocimiento se difunda en todos los sectores y se pueda potenciar. Es innegable el papel del talento humano en este proceso. Son los integrantes de la organización quien en últimas se apropian del conocimiento, lo utilizan, analizan sus impactos y hacen mejoras para luego transferir resultados a la sociedad (Karanikola y Panagiotopoulos, 2018).

De tal manera, la comunidad mundial ha emprendido una transformación primordial al desarrollar desde la sociedad industrial a la sociedad de la información; según Azpiazu, Pazos, y Silva (2001), la educación y la sapiencia se constituyen en propulsores de esta sociedad. No obstante, según Alfonso (2016) en la manifestación "sociedad de la información" se identifica el valor social que se le confiere a la comunicación y la información en la colectividad actual, donde se comprometen las relaciones económicas, culturales y sociales.

Ahora bien, las universidades y centros de capacitación deben ser conscientes del papel determinante que juegan para hacer del manejo adecuado de las tecnologías de la información y la comunicación una competencia en la formación de sus estudiantes. Estos resultados se pueden obtener con la aplicación de estrategias como el aprendizaje por problemas o el estudio de casos, de esta forma no solo se aprende lo teórico, sino que se transfiere a la práctica (López-Lemus y De la Garza Carranza, 2018).

La cuarta revolución industrial está impulsada por la transformación digital, lo cual implica ciertas ventajas competitivas como optimización de los procesos, economías dinámicas, adecuada toma de decisiones, entre otras. Sin embargo, pese a los cambios y evolución que ha experimentado la industria en cada una de sus fases, especialmente en la actualidad, el factor humano ha permanecido constante (González, 2018).

De igual manera, la Cuarta Revolución brinda nuevas posibilidades de desarrollo económico, social y personal, pero puede llevar a la exclusión de personas, aumentar la desigualdad y obstaculizar las relaciones personales 
(UNESCO, 2018). La inteligencia artificial imita las funciones de los seres humanos. Sin embargo, no posee sus características diferenciales como la creatividad, la inteligencia emocional, la intuición y el liderazgo tan necesarios en el ámbito laboral (González, 2018).

En este sentido, la investigación desarrollada por ManpowerGroup (2018), en el que aplicó un cuestionario en 44 países, permitió demostrar que la confianza en la automatización ha ido creciendo pues no han disminuido las oportunidades de empleo. Las empresas están gestionando el conocimiento dentro de la organización para que los empleados desarrollen otras funciones. Se afirma, además, que las empresas se están convirtiendo en "Constructoras de talento", la investigación también afirma que 77\% de las empresas quiere desarrollar nuevas competencias en el talento humano.

No obstante, a medida que avanzamos hacia las organizaciones 4.0, se hace necesario considerar que, si bien los humanos aceptan el cambio, no funcionan bien en posiciones inestables. Es necesario en un ambiente cambiante y complejo crear la estabilidad laboral que la persona necesita. Esto será un nuevo reto debido a que se anticipa que las fuerzas de trabajo tendrán una tendencia hacia empleos temporales, por lo tanto, los empleados deben gestionar el conocimiento y adquirir las competencias y habilidades que el mercado necesita (Deloitte Insights, 2018).

De acuerdo con Ranz (2016), el Talento 4.0 lo compone aquellas nuevas capacidades y habilidades relacionadas con nueva tecnología que adquieren las personas en pro de apoyar a la empresa a implementar estrategias que la encaminan en la industria 4.0. Debido a lo anterior, el Talento 4.0 es inevitable para llegar a la cuarta revolución industrial, por ello es fundamental la educación, debido a que solo ella podrá promover el talento que se necesita (Ver figura 9).

Figura 9

Educación 4.0

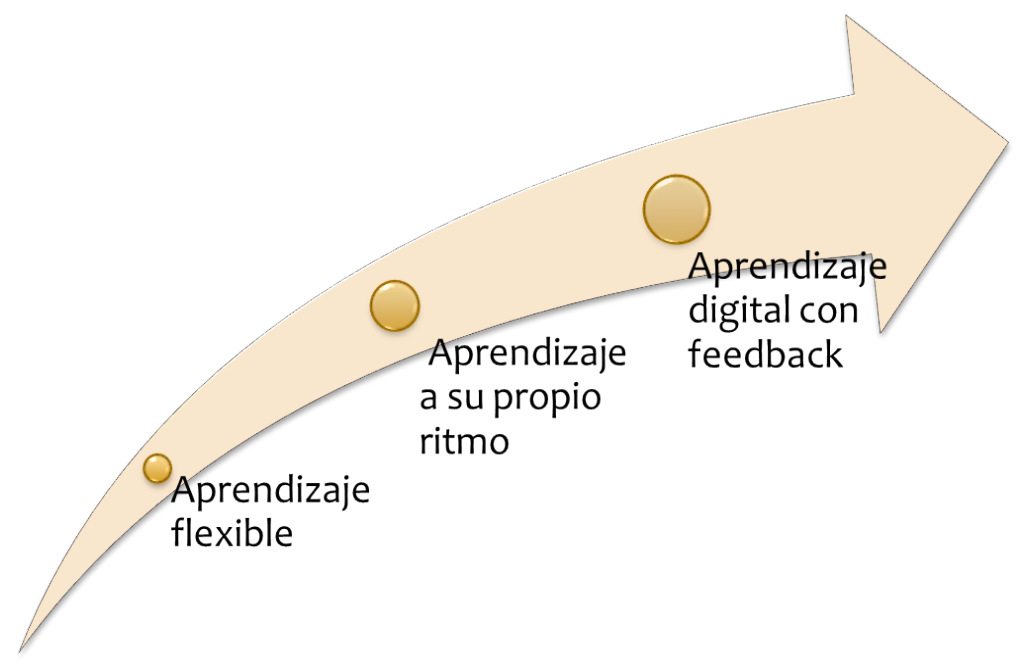

Así mismo, el NMC Horizon Report para la educación superior plantea algunas tendencias como el avance de la cultura de adaptación al cambio e innovación, reingeniería de los lugares de aprendizaje y cambio de los enfoques de aprendizaje a unos más especializados, desafíos significativos como la personalización del aprendizaje, conservar la importancia de la educación y por último, algunos desarrollos importantes en la tecnología educativa para la educación 4.0 como la realidad virtual, Makerspaces y robótica (Ranz, 2016).

Cabe agregar, que la transformación digital va impactando cada vez más a las organizaciones y sobre todo al talento humano. El mundo laboral ha evolucionado enormemente lo cual constituye un desafío para los que trabajan en esta área, ya que tienen que fortalecer su perfil profesional estando encaminado en la nueva era de 
la tecnología. Por ejemplo, un estudio que se realizó en Estados Unidos dio a conocer un dato interesante: Más de la mitad de las profesiones importantes en la actualidad no existirán para el año 2030, debido a la evolución que ha venido tomando el talento humano (Abascal, 2017).

Por lo tanto, es necesario recalcar que estas nuevas competencias requeridas en la industria 4.0 permitirán crear nuevos empleos, pero en igual medida algunos trabajadores serán desplazados. Esto significa que es necesaria la formación y el desarrollo de nuevas destrezas y habilidades para enfrentar los nuevos retos y por ende mejorar su calidad de vida. Entre todos los desarrollos, desafíos y tendencias el que más va a generar impacto será el uso de las analíticas de datos, porque todos estos servicios de aprendizaje están desarrollando algoritmos que comprueban el aprendizaje progresivo de los estudiantes (Ranz, 2016). Por ello, la informática y sus profesionales serán elementos clave en el escenario de industria 4.0 que permita a la industria mejorar su competitividad (Del Val Roman, 2016).

De acuerdo con la investigación de Amaya (2019), el recurso humano ha tenido cierta evolución gracias a la industria 4.0. La transformación de esta se ha dado en la estructura interna de la gestión del recurso humano, la contratación, atracción, reclutamiento, rendimiento y planificación.

De igual forma, Platas (2017) afirma que la entrada de la cuarta revolución ha traído diversos cambios, pero no solo en la forma de administrar a las empresas sino también la gestión del recurso humano. Un ejemplo claro donde se aprecia el impacto de las TIC en la administración del recurso humano es la nueva forma de reclutamiento de personal. En los años noventa apareció lo que es el primer portal de empleo vía web, donde se recibían hojas de vida por fax o correo. En aproximadamente 20 años se pasa del reclutamiento 1.0 a reclutamiento 4.0 (ver figura 10 ).

Figura 10

Reclutamiento 4.0

\begin{tabular}{|l|l|}
\hline 1.0 & $\begin{array}{c}\text { - Contratación tradicional mediante fax y publicidad } \\
\text { impresa }\end{array}$ \\
\hline 2.0 & $\begin{array}{c}\text { - Tecnología en línea mediante bolsas de trabajo y hojas de } \\
\text { vida en línea }\end{array}$ \\
\hline 3.0 & $\begin{array}{r}\text { - Atracción del mejor talento a través de marca empleadora, } \\
\text { marketing digital y comunicacion bidireccional }\end{array}$ \\
\hline 4.0 & $\begin{array}{c}\text { - Se contrata a través de la nube, crowdsourcing, } \\
\text { aplicaciones móviles y gamificación }\end{array}$ \\
\hline
\end{tabular}

Fuente: Los autores a partir de Platas (2017)

Las personas tienden a fijarse en organizaciones donde la innovación y la tecnología se aplican ya que tienden a generar un buen desarrollo en el área del personal, en el sentido de atraer, incorporar, motivar, desarrollar y retener a aquellos profesionales que permitan cumplir los objetivos de la empresa a largo plazo (Platas, 2017). La introducción de la revolución industrial trae consigo grandes cambios en los procesos organizacionales debido a la aplicación de los elementos que la componen, como el internet de las cosas, el big data, la nube, la inteligencia artificial, entre otros. La industria 4.0 también afecta directamente la forma de gestionar a las personas, por ello se debe identificar estos cambios y analizar las respuestas de las organizaciones con respecto a este nuevo desafío (Amaya, 2019). 
Por su parte, Marnewick y Marnewick (2019) confirman que se debe reconocer que en el futuro el talento humano será un "ecosistema híbrido" donde los individuos y la máquina tendrán redes de aprendizaje que permitirán una adecuada gestión del conocimiento dentro de la organización. No obstante, Shamim, Cang, Yu y Li, (2016) persisten en la importancia de un ambiente de aprendizaje e innovación dentro de la organización.

Debido a esto, Jeschke,Brecher, Meisen, Özdemir y Eschert (2017) consideran que en un sistema ciberfísico es hacia dónde va la tendencia en la gestión del talento humano. Es decir, la cibernética, ingeniería mecánica, electrónica, mecatrónica, industrial y las tecnologías emergentes tienen que estar alineadas con el recurso humano.

Como resultado, se puede afirmar que la aparición repentina de las nuevas tecnologías ha venido transformando a la sociedad y la economía (González, Peñaranda y Manzano, 2019). Pero mucho más la gestión del talento humano, en el sentido de que existen cambios como el uso de las tecnologías emergentes, las nuevas formas de organización como lo son el teletrabajo y la implementación de nuevos y mejores procesos de mayor calidad que han impactado a las organizaciones y por lo tanto se hace necesario generar en las empresas y en los centros educativos una cultura de cambio para tomar la transformación digital como una oportunidad de mejora en todos los ámbitos organizacionales.

En este sentido, para desarrollar las competencias que se requieren (ver Figura 11) en la sociedad y la organización 4.0 es necesario impulsar 8 tipos de pensamientos enfocados en los conocimientos que requiere para desempeñarse en forma efectiva como parte activa de la sociedad y la organización 4.0.

En este sentido, las personas con Talento 4.0 son aquellas que poseen competencias y aptitudes relacionadas con la Industria 4.0, siendo el producto de la combinación de aptitudes personales con la experiencia y la formación. Por lo tanto, en la figura 12 se incluyen los conocimientos que requieren para desempeñarse en forma efectiva en la organización 4.0 
Figura 11

Competencias requeridas en la sociedad y organización 4.0

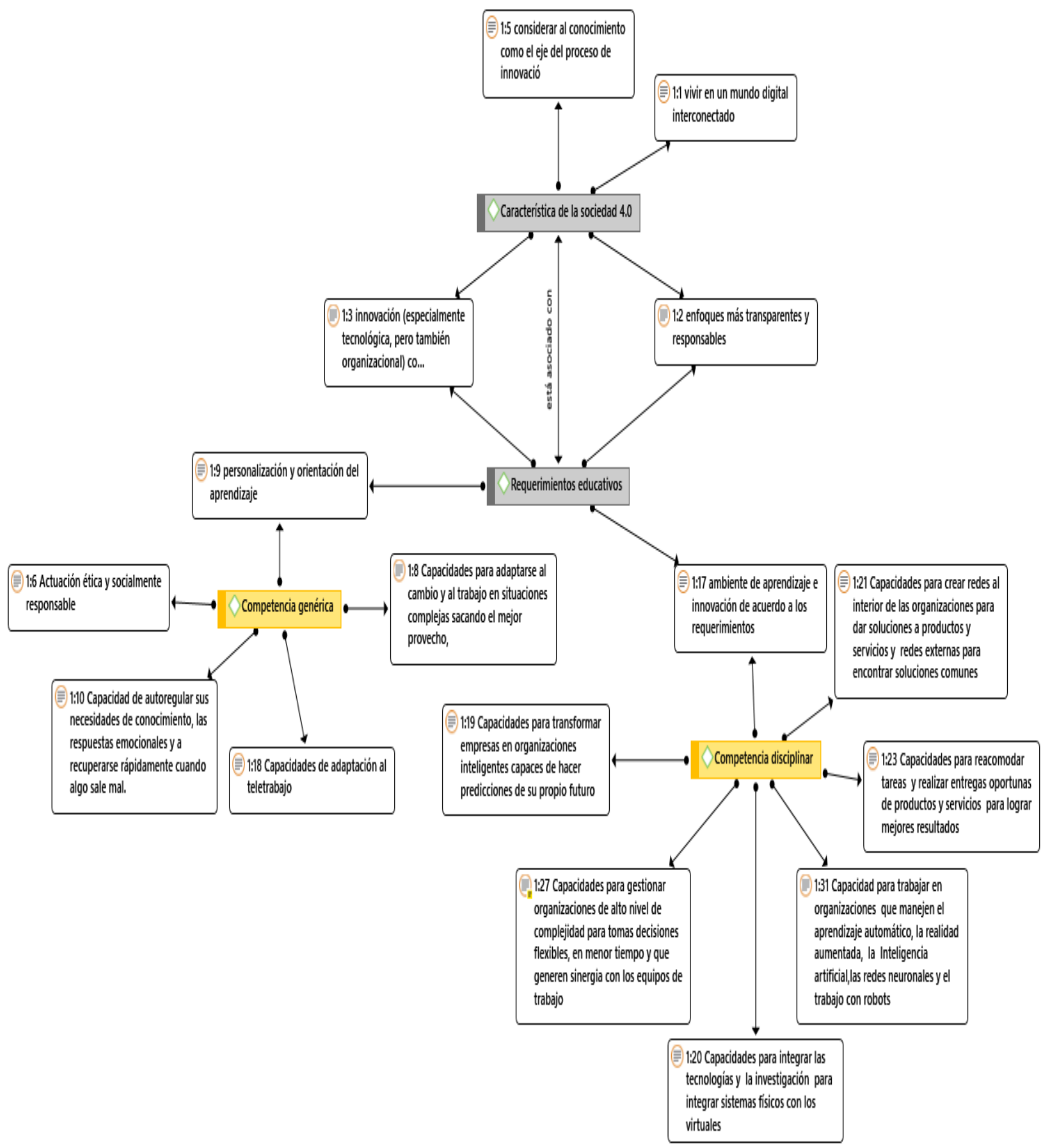

Fuente: Los autores 
Figura 12

Conocimientos del Talento humano 4.0

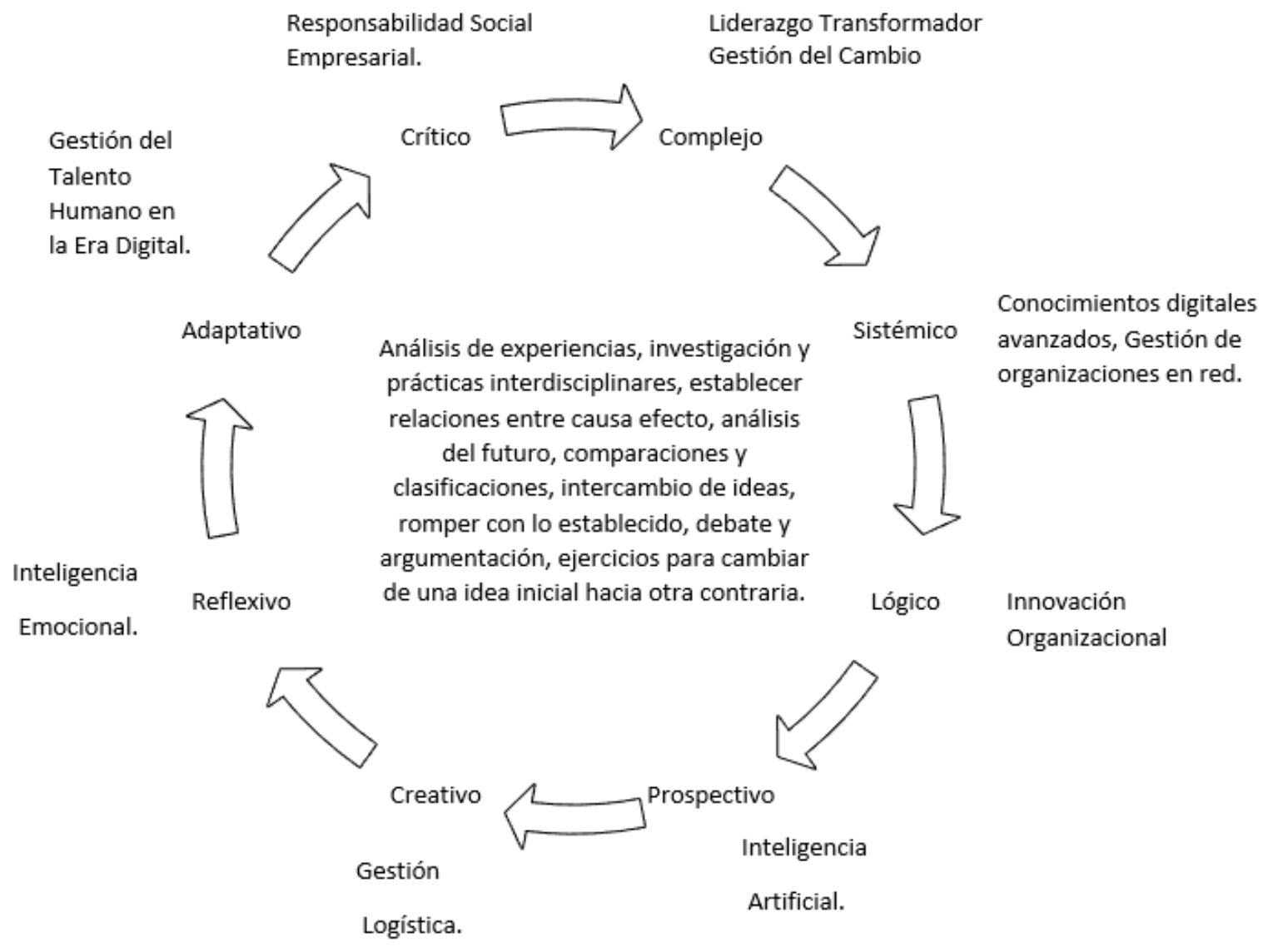

Fuente: Los autores a partir de la bibliografía citada.

\section{Conclusiones}

Una sociedad que valora la importancia de la información como una clave para la riqueza económica y la prosperidad y donde hay un aumento en las actividades relacionadas con la misma, se reconocerá una mejora del capital humano y su capacidad intelectual. La sociedad 4.0 garantiza la libertad de información mediante el uso de tecnologías emergentes, donde se puede intercambiar conocimiento a nivel local, regional y global.

De la bibliometría se puede evidenciar cómo la industria u organización 4.0 se ha venido desarrollando en forma vertiginosa en los últimos años y la difusión del conocimiento se materializa utilizando ponencias y artículos de rápida difusión por la acelerada innovación que este tema representa. En el mapa tecnológico efectuado con software VOSViewer, se evidencian 8 clúster, cada uno de ellos representa los elementos fundantes de la organización 4.0 y son: Innovación, logística, comunicación, internet de las cosas, sistemas automáticos y robótica, producción, evaluación y control, nuevas tecnologías en sistemas de información y analítica de datos.

Se concluye que para desarrollar las competencias que se requieren en la sociedad y la organización 4.0 es necesario promover siguientes conocimientos en: Gestión de organizaciones en red, Liderazgo transformador, Inteligencia Artificial, Innovación Organizacional, Gestión Logística, Gestión del cambio, Inteligencia emocional, Gestión del talento humano en la era digital, Responsabilidad social empresarial y Conocimientos digitales avanzados. 


\section{Referencias bibliográficas}

Abascal, I. (2017). Profesionales 4.0, el talento que viene. RRHHDigital. Recuperado de http://www.rrhhdigital.com/editorial/128155/Profesionales-40-el-talento-que-viene-

Alfonso Sánchez, I. (2016). The Information Society, Knowledge Society and Learning Society. Referring to their training. 12 (2), 235-243.

Amaya, R. (2019). La revolución industrial 4.0: transformaciones en las organizaciones y la gestión humana en el periodo 2015 - 2019 (tesis de pregrado). Universidad Piloto de Colombia, Bogotá, Colombia.

Recuperado de:

http://repository.unipiloto.edu.co/bitstream/handle/20.500.12277/6120/Ricardo\%20\%20Amaya\%20LA\%2 OREVOLUCION\%20\%204072019.pdf?sequence=1\&isAllowed=y

Amor Bravo, E. M. (2019). El marketing y la cuarta revolución indutrial. Madrid: ESIC Editorial.

Avendaño, W. (2012). Innovación: un proceso necesario para las pequeñas y medianas empresas del municipio de San José de Cúcuta, Norte de Santander (Colombia). Semestre Económico, 15(31), 187-207.

Azpiazu, J., Pazos, J. y Silva, A. (2001). La teleformación mediante Internet. En Actas de El futuro de Internet. Acceso y Teleservicios, España: Fundación Alfredo Brañas.

Bustillo-García, L. y Martínez-Dávila, J. P.(2008). Los enfoques del desarrollo sustentable. Interciencia, 33(5), 389-395.

Chandrasekar, G., y Sharma, R. (2010). Analyzing knowledge disparity and value creation: Towards a k-gini coefficient. International Journal of Knowledge-Based Development, 1(3), 242-262.

Del Val Roman, J. (2016). Industria 4.0: La transformación digital de la industria. Coddiinforme, 1-19. Recuperado de http://coddii.org/wp-content/uploads/2016/10/Informe-CODDII-Industria-4.0.pdf

Deloitte Insights (2018). La cuarta revolución industrial está aquí - ¿Está usted preparado? Recuperado de https://www2.deloitte.com/content/dam/Deloitte/co/Documents/aboutdeloitte/Revoluci\%C3\%B3n\%204.0\%20142201.pdf

Díaz, J., Pérez, A y Florido, R. (2011). Impacto de las tecnologías de la información y las comunicaciones (TIC) para disminuir la brecha digital en la sociedad actual. Cultivos Tropicales, 32(1), 81-90.

Díaz-Martínez, M.A., Cruz-Méndez, A.L., y Ruiz-Domínguez, H.S.. (2018). Instrumento de diagnóstico y autoevaluación para medir las condiciones organizacionales hacia la nueva revolución industrial 4.0. RIIIT. Revista internacional de investigación e innovación tecnológica, 6(35), 1-14.

Domínguez Sánchez-Pinilla, Mario (2003). Las tecnologias de la informacion y la comunicación: sus opciones, sus limitaciones y sus efectos en la enseñanza. Nómadas. Critical Journal of Social and Juridical Sciences, (8). Recuperado de: https://www.redalyc.org/articulo.oa?id=181/18100809

Drath R. y Horch, A. (2014). Industrie 4.0: hit or hype, IEE Ind. Electron. Mag. 8 (2), 56-58.

Fonseca, L., Ramos, A., Rosa, A., Braga, A.C. y Sampaio, P. (2016). Satisfacción de las partes interesadas y éxito sostenible. Revista Internacional de Ingeniería Industrial y de Sistemas, 2 (2), 144-157.

García, G. (2018). Máquinas, robots y economía circular. Revista Telos: Tecnoética. Recuperado de: https://telos.fundaciontelefonica.com/revista/telos-109/ 
González, Y. Peñaranda. M y Manzano, O. (2019). Innovaciones tecnológicas en las prácticas académicas Virtuales. Revista Colombiana de Tecnologías de Avanzada, 1 (33), 69-77

González, N. (2018). Factor Humano 4.0: Innovación Centrada en las Personas para la Industria 4.0 [Mensaje en un blog]. Recuperado de http://prevenblog.com/factor-humano-4-0-innovacion-centrada-las-personas-laindustria-4-0/

Hernández, R. Fernández C. y Baptista L. (2014). Metodología de la investigación. México: Mc Graw Hill interamericana editores.

Heidenreich, M. (2003). Die Debatte um die Wissensgesellschaft, In S. Böschen and I. Schulz-Schaeffer (Eds.). Wissenschaft in der Wissensgesellschaft, pp. 25-51. Opladen: Westdeutscher Verlag.

Jamett, I, Alvarado, L, y Maturana, S. (2017). Análisis al estado del arte de la innovación abierta: Implicaciones prácticas en la ingeniería. Revista ingeniería de construcción, 32(2), 73-84.

Jeschke, S., Brecher, C., Meisen, T., Özdemir, D., y Eschert, T. (2017). Industrial internet of things and cyber manufacturing systems. In Industrial Internet of Things (pp. 3-19). Springer, Cham International Publishing.

Kagermann, H.; Wahlster, W. y Helbig, J. (2013). Recommendations for implementing the strategic initiative industrie 4.0. Acatech, 1-82.

Karanikola, Z., y Panagiotopoulos, G. (2018). 4a revolución industrial: el desafío de cambiar las habilidades de los recursos humanos. Revista Europea de Formación y Desarrollo, 5 (3), 1-7

Khan, A. W. (2003). Hacia las sociedades del conocimiento. An Interview with Abdul Waheed Khan, World of Science, 1 (4), UNESCO's Natural Sciences Sector.

Kozma, R. y Schank, P. (2000). Conexión con el siglo XXI: la tecnología como soporte de la reforma educativa. En D. Chris. Aprendiendo con Tecnología. Argentina: Editorial Paidós SAICF

Lee, J. (2014). La Industria 4.0: la fábrica en el entorno de los macrodatos", tec.News - Boletín tecnológico de Harting, 26, 8-9.

López-Lemus, J. y De la Garza Carranza, M. (2018). Tecnología y aprendizaje organizacional factores influyentes sobre la satisfacción profesional en residentes de pregrado. Actualidades Investigativas en Educación, $18(2), 285-308$.

López, M., Lovato, S. y Abad, G.(2018). El impacto de la cuarta revolución industrial en las relaciones sociales y productivas de la industria del plástico IMPLASTIC S. A. en Guayaquil-Ecuador: retos y perspectivas. Revista Universidad y Sociedad, 10(5), 153-160.

ManPowerGroup. (2018). Revolución de habilidades 4.0. Recuperado de https://www.manpowergroup.com.mx/wps/wcm/connect/manpowergroup/4b4e85e9-e77d-4226-8b1edc8eb968b5c5/MPG_WEF_SkillsRevolution_4.0_MXb.pdf?MOD=AJPERES\&CONVERT_TO=urI\&CACHEID=4b4e85e9-e77d-4226-8b1e-dc8eb968b5c5

Marnewick, C. y Marnewick, A.(2019). The Demands of Industry 4.0 on Project Teams. IEEE Transactions on Engineering Management, 67(3). DOI: 10.1109/TEM.2019.2899350 
Molina, A., Roque, L., Garcés, B., Rojas, Y., Dulzaides, M y Selín, M. (2015). El proceso de comunicación mediado por las tecnologías de la información. Ventajas y desventajas en diferentes esferas de la vida social. MediSur, 13(4), 481-493.

Pérez, O. (2018). Caminando hacia la Industria 4.0: blockchain, Al, robótica y loT. Recuperado de: https://planetachatbot.com/caminando-hacia-la-industria-4-0-blockchain-ai-rob\%C3\%B3tica-y-iotca0f497e95d3

Pesce, F., Rigon, V., Tregnaghi y Voglino, A. (2017). An Industry 4.0 case study in fashion manufacturing. ELSEVIER, 11, 871-877.

Platas, V. (2017). Digitalización de la Gestión de personas. Oikonomics revista de Economía, Empresa y Sociedad, (8), 81-89. Recuperado de:

http://oikonomics.uoc.edu/divulgacio/oikonomics/_recursos/documents/08/7_Platas_Oikonomics_8_cast _a4.pdf

Ranz, R. (30 de mayo del 2016). Una educación 4.0 para el fomento del talento 4.0 [Mensaje de un blog]. Recuperado de https://robertoranz.com/2016/05/30/una-educacion-4-0-para-el-fomento-del-talento-4-0/

Restrepo, C. (2013). Aproximación a la gestión de la innovación: un enfoque conceptual. Revista Ciencias Estratégicas, 22(30), 257-266.

Rueda-López, J. (2007). La tecnología en la sociedad del siglo XXI: albores de una nueva revolución industrial. Aposta. Revista de Ciencias Sociales, 1(32), 1-28.

Shamim, S., Cang, S., Yu, H., y Li, Y. (2016). Management approaches for Industry 4.0: A human resource management perspective. In 2016 IEEE Congress on Evolutionary Computation (CEC) (pp. 5309-5316). IEEE.

UNESCO. (2018). Políticas públicas para la innovación 4.0. Recuperado de http://www.unesco.org/new/fileadmin/MULTIMEDIA/FIELD/Mexico/images/Publicaciones/Agendalnnova cion402C.pdf

Weingart, P. (2001). Die Stunde der Wahrheit? Zum Verhältnis der Wissenschaft Politik, Wirtschaft und Medien in der Wissensgesellschaft. Weilerswist: Velbrück.

Yigitcanlar, T. (2011). Position paper: Redefining knowledge-based urban development. International journal of Knowledge-Based Development, 2(4), 340-356.

Ynzunza, C., Izar, J., Bocarando, J., Aguilar, F., y Larios, M. (2017). El Entorno de la Industria 4.0: Implicaciones y Perspectivas Futuras. Conciencia tecnológica, (54), 1-23. Recuperado de http://www.redalyc.org/jatsRepo/944/94454631006/index.html

Esta obra está bajo una Licencia Creative Commons

Attribución-NoCommercial 4.0 International

\section{(cc) EY-NC}

